\title{
The effects of intramuscular and intraperitoneal injections of benzo(a)pyrene on selected biomarkers in Clarias gariepinus.
}

\begin{abstract}
This study investigated the dose-dependent and time-course effects of intramuscular (i.m.) and intraperitoneal (i.p.) injection of benzo[a]pyrene (BaP) on the biomarkers EROD activity, GST activity, concentrations of BaP metabolites in bile, and visceral fat deposits (Lipid Somatic Index, LSI) in African catfish (Clarias gariepinus). Intraperitoneal injection resulted in 4.5 times higher accumulation of total selected biliary FACs than i.m. injection. Hepatic GST activities were inhibited by $\mathrm{BaP}$ via both injection methods. Dose-response relationships between $\mathrm{BaP}$ injection and both biliary FAC concentrations and hepatic GST activities were linear in the i.p. injected group but nonlinear in the i.m. injected fish. Hepatic EROD activity and LSI were not significantly affected by BaP exposure by either injection route. We conclude that i.p. is a more effective route of exposure than i.m. for future ecotoxicological studies of PAH exposure in C. gariepinus.
\end{abstract}

Keyword: Benzo(a)pyrene; Biomarkers; Polyaromatic hydrocarbon. 